\title{
Outcome of One and Two Visit Root Canal Treatment of Irreversible Pulpitis
}

\author{
Md. Abdur Rahim, ${ }^{7}$ Md. Nurul Amin, ${ }^{2}$ Khurshid Mahmood, ${ }^{3}$ Sibbir Ahmed Osmani, ${ }^{4}$ Abul Kalam Md. Faruq, ${ }^{5}$
}

\begin{abstract}
Objective: The present prospective comparative clinical trial was undertaken to compare the outcomes of single and two-visit root canal treatment of irreversible pulpitis.

Methodology: This prospective study was conducted in the Department of Conservative Dentistry and Endodontics of Dhaka Dental College on patients who presented with pain due to irreversible pulpitis. Patients with single-rooted teeth with uncomplicated canal and full-formed apex having irreversible pulpitis were included. However, pregnant women or patients under treatment of antibiotics, cortiosteiods or suffering from any systemic diseases or immuno-compromised patients or patients having teeth with calcified canal or periodontal disease were excluded. A total of 80 patients who met the eligibility criteria were consecutively included and were randomly assigned to either one-visit $(n=40)$ or two-visit $(n=40)$ treatment groups. Data were kept on pulp vitality status, the presence or absence of pre-operative pain and degree of post obturation pain at $6,12,24,48$ hours and 1 week postoperatively. The outcome variable was intensity of pain which was measured using a visual analogue scale (VAS) as $0=$ no pain, $1=$ slight pain or discomfort, $2=$ moderate pain relieved by analgesic, $3=$ moderate to severe pain not completely relieved by analgesics and $4=$ severe pain or swelling not relieved by analgesics and required unscheduled visit.

Result: Forty percent of the patients in the two-visit group did not have any pain (measured in terms of visual analogue scale) at 12 hours compared to $25 \%$ in the single visit group, although the difference did not turn significant $(p=0.580)$. Likewise the proportion of patients without pain in the two-visit group was considerably higher $(52.5 \%)$ at 24 hours than that in the single-visit group $(45 \%)(p=0.852)$. Improvement in pain sensation was reported to be significantly higher in the two-visit group (as $95 \%$ of the patients did not complain any pain) at 48 hours than that in the single-visit group ( $80 \%$ of the patients did not complain any pain) $(p=$ 0.015). None of the patients of either group complained of pain one week postoperatively.

Conclusion: Compared to single-visit, two-visit root canal treatment showed better results in terms of intensity of post-obturation pain at 12, 24 and 48 hours after root canal treatment for irreversible pulpitis.

Key words: Irreversible pulpitis, one-visit and two-visit root canal treatment, intensiti etc.
\end{abstract}

\section{INTRODUCTION}

Pain management is one of the more challenging aspects of the clinical practice of endodontics. Irreversible pulpitis at an advanced stage of development is characterized by acute and intense pain that is difficult to control with pain killers. ${ }^{1,2}$ Hence the patient needs urgent endodontic help. In such cases, quick and precise diagnosis is

\section{Authors' information:}

'Dr. Md Abdur Rahim, BDS, MS (Conservative Dentistry \& Endodontics), Assistant Professor, Department of Conservative Dentistry \& Endodontics, Dhaka Dental College, Dhaka.

${ }^{2}$ Dr. Md. Nurul Amin, MBBS, DMCH, M. Phil (PSM), MPH (Thailand) Assistant Professor Community Medcine, Rajshahi Medical College, Rajshahi.

${ }^{3}$ Dr. Khurshid Mahmood, MBBS, FCPS (Physical Medicine), Associate Professor and Head, Department of Physical Medicine, National Institute of Neuroscience, Dhaka.

${ }^{4}$ Dr. Sibbir Ahmed Osmani, BDS, MS (Prosthodontics), Associate Professor \& Vice Principal, Dhaka Dental College, Dhaka.

${ }^{5}$ Dr. Abul Kalam Md. Faruq, BDS, MS (Oral \& Maxillofacial Surgery), Assistant Professor, Department of Oral \& Maxillofacial Surgery, Dhaka Dental College, Dhaka.

Correspondence: Dr. Md Abdur Rahim, Cell phone: +880 17153392116, E-mail: rahimdental68@gmail.com 
necessary for immediate patient management. ${ }^{3-6}$ Often the lack of time becomes a factor that significantly influences the treatment protocol.

Single- versus multiple-visit root canal treatment has been the subject of long-standing debate in the endodontic community. In fact the attempt to complete root canal treatment in one-visit has been documented since the end of the $19^{\text {th }}$ century. ${ }^{7}$ A one-visit root canal treatment is attractive to patients because it saves time and would probably reduce the cost of the procedure. Additionally one-visit treatment is expected to be less stressful to anxious patients. Patients are not disturbed by additional anesthetic injections, isolation, the initial placement and later removal of intracanal medication and seals, and the time spent by the clinician in refreshing his memory and tactile sensation regarding prepared canal anatomy, tooth length etc. Furthermore, the problems of inter-visit leakage, loss of temporary seal or any of the accidents that may occur between the visits could be solved. Perhaps the most important advantage of one-visit treatment is the prevention of root canal contamination and/or bacterial re-growth that may result when the treatment is prolonged over an extended period. ${ }^{8}$ However, the single visit procedure has its demerits as well. It eliminates some of the controls, available in the multiple visit procedure, such as culturing to check the effectiveness of the bio-mechanical preparation, ability to apply "tincture of time" to re-evaluate tissue responses following treatment procedures and in the event of flare up, the emergency procedures for drainage are complicated, since artificial fistualation or the removal of filling material is needed. ${ }^{9}$

The present study has been designed to determine the minimum set of interventions needed in order to reduce the pain and prevent further complications when treating emergency cases of irreversible pulpitis.

\section{METHODOLOGY}

This prospective study was conducted in the Department of Conservative Dentistry and Endodontics of Dhaka Dental College on individuals who presented with pain due to irreversible pulpitis of single-rooted teeth of either upper or lower jaw. Patients with single-rooted teeth with uncomplicated canal and full-formed apex having irreversible pulpitis were eligible for inclusion into the study. However, pregnant women or patients under treatment of antibiotics, corticosteroids or suffering from any systemic diseases or immuno-compromised patients were excluded from the study. Patients having teeth with calcified canal (evaluated by periapical radiograph), periodontal disease or periapical radiolucency were also excluded. Based these enrolment criteria, a total of 80 patients (male 49, female 31 ) aged 20 to 50 years were consecutively included in the study. Patients were randomly assigned to either one- or two-visit treatment group-40 patients received one-visit treatment and 40 patients two-visit treatment. After having verbal consent from the patients, data were collected on variables of interest (age, gender, tooth location and pulpitis diagnosis) using a semi-structured questionnaire.

The diagnosis was made using data from the anamnesis, objective clinical studies (examination, probing, percussion, palpation) and X-ray investigation. The predominant symptom in the anamnesis was spontaneous and continuing pain, recurring periodically and not responding to peripheral analgesics. Examination revealed big carious defects with impaired functioning of the teeth. The X-ray studies showed developing carious process near the tooth pulp and no evidence of periapical changes. For each tooth treated data were kept on pulp vitality status, the presence or absence of pre-operative pain and degree of post obturation pain at $6,12,24,48$ hours and 1 week postoperatively. The intensity of pain was measured using a visual analogue scale (VAS). Patients were contacted over telephone at $6,12,24,48$ hours and 1 week to remind them to complete the VAS form and to return the same 7 days after completion of root canal treatment.

The standard procedure for both groups at first appointment included local anaesthesia (L/A), 
isolation, caries excavation and standard access preparation. The working length was determined radio graphically from a coronal reference to a distance $1 \mathrm{~mm}$ short of radiographic apex. The root canal were cleaned and shaped using step back technique.

For single-visit group, chemico-mechanical preparation was done. Each root canal was dried with paper points and was filled with gutta-percha points sealed with zinc oxide eugenol sealer using lateral condensation technique. For two-visit group, teeth were prepared as in the former group but were not obturated. Chemico-mechanical preparation was done in the first visit using the same technique for all cases. A sterile cotton pellet was placed in the pulp chamber and the access cavity was sealed with Zno. eugenol cement. One week later the teeth were obdurated as of single-visit group. All patients were prescribed 50 mg of diclofenac sodium (to be taken only if they experience moderate pain). Any of the patients with intolerable pain were requested to visit for emergency treatment. The evaluation of post obturation pain was done with the visual analogue scale (VAS). The post operative evaluation was recorded as: $0-$ no pain, 1 -slight pain or discomfort, 2-moderate pain relieved by analgesic, 3-moderate to severe pain not completely relieved by analgesics and 4 - severe pain or swelling not relieved by analgesics and required unscheduled visit.

\section{RESULT}

Forty percent of the patients in the two-visit group did not have any pain (measured in terms of visual) at 12 hours postpperatively compared to $25 \%$ in the single visit group, although the difference did not turn significant $(p=0.580)$ (Table I). Likewise the proportion of patients without pain in the two-visit group after 24 hours was considerably higher $(52.5 \%)$ than that in the single-visit group $(45 \%)(p=0.852)$ (Table II). Improvement in pain sensation was reported to be significantly higher in the two-visit group (as 95\% of the patients did not complain of any pain) at 48 hours than that in the single-visit group $(80 \%$ of the patients did not complain any pain) $(\mathrm{p}=$ 0.015 ) (Table III). None of the patients of either group complained of any pain one week postoperatively.

\begin{tabular}{l|ccc|}
\hline $\begin{array}{l}\text { TABLE I. Comparison of pain intensity between groups at } \\
\text { 12 hours postoperatively }\end{array}$ & \multicolumn{3}{c}{ Group } \\
\\
Intensity of pain (VAS) & $\begin{array}{c}\text { Single-visit } \\
(\mathrm{n}=40)\end{array}$ & $\begin{array}{c}\text { Two-visit } \\
(\mathrm{n}=40)\end{array}$ & p-value \\
\hline 0 & $10(25.0)$ & $16(40.0)$ & \\
1 & $14(35.0)$ & $12(30.0)$ & \\
2 & $8(20.0)$ & $8(20.0)$ & 0.580 \\
3 & $6(15.0)$ & $3(7.5)$ & \\
4 & $2(5.0)$ & $1(2.50$ & \\
\hline
\end{tabular}

TABLE II. Comparison of pain intensity between groups at 24 hours postoperatively

\begin{tabular}{lccc} 
Intensity of pain (VAS) & \multicolumn{2}{c}{ Group } & \\
& $\begin{array}{c}\text { Single-visit } \\
(\mathrm{n}=40)\end{array}$ & $\begin{array}{c}\text { Two-visit } \\
(\mathrm{n}=40)\end{array}$ & p-value \\
0 & $18(45.0)$ & $21(52.5)$ & \\
1 & $11(27.5)$ & $10(25.0)$ & \\
2 & $7(17.5)$ & $6(15.0)$ & 0.852 \\
3 & $3(7.5)$ & $3(7.5)$ & \\
4 & $1(2.5)$ & $0(0.0)$ &
\end{tabular}

\begin{tabular}{|c|c|c|c|}
\hline \multirow[b]{2}{*}{ Intensity of pain (VAS) } & \multicolumn{2}{|c|}{ Group } & \multirow[b]{2}{*}{ p-value } \\
\hline & $\begin{array}{l}\text { Single-visit } \\
\quad(n=40)\end{array}$ & $\begin{array}{l}\text { Two-visit } \\
(n=40)\end{array}$ & \\
\hline 0 & $32(80.0)$ & $38(95.0)$ & \multirow{5}{*}{0.015} \\
\hline 1 & $6(15.0)$ & $2(5.0)$ & \\
\hline 2 & $2(5.0)$ & $0(0.0)$ & \\
\hline 3 & $0(0.0)$ & $0(0.0)$ & \\
\hline 4 & $0(0.0)$ & $0(0.0)$ & \\
\hline
\end{tabular}

\section{DISCUSSION}

There is ongoing debate among researchers and clinician concerning the effectiveness of a single-visit versus multiple-visit endodontic treatment in terms of post-obturation pain. ${ }^{10}$ Root-canal treatment with irreversible pulpitis is reported to be associated with higher incidence and intensity of pain of post obturation at 6,12 , 24, 48 hours. As all patients, in the present study, were under cover of analgesics for first 6 hours, pain sensation was not evaluated before 12 hours, 
but two visit-root canal treatment showed better result in terms of post obturation pain management after 12, 24 and 48 hours. Consistent with these findings, Figini et al. ${ }^{11}$ reported patients undergoing single-visit root canal treatment may experience slightly higher frequency of post obturation pain compared to their two-visit counterparts and is more likely to take pain killers. ${ }^{11-14}$

In contrast, Modaresi et al ${ }^{15}$ found low incidence of post obturation pain in single-visit endodontic treatment as compared to two visit endodontic treatment, which has also been supported by Albashaireh et $\mathrm{al}^{16}$ who found a significantly higher intensity of post obturation pain in the multiple visit within 24 hours of obturation. The reason for the higher incidence and severity of post-operative pain after treatment of teeth with irreversible pulpitis is not completely clear. One possibility is that the injury of periapical vital tissue during endodontic treatment in teeth with vital pulp promotes more intensive secretion of inflammatory mediators like prostaglandins, serotonin, histamine and bradykinin (all of which are also pain mediators).

The limitation of this study was that VAS had been used to measure the levels of pain and the pain experience is very subjective and is dependent on so many factors. However, a careful case selection and adherence to the basic principles of endodontic treatment will reduce the incidence of post obturation pain and will increase the incidence of successful outcome.

\section{CONCLUSION}

Compared to single-visit, two-visit root canal treatment showed better results in the terms intensity of post obturation pain after 12, 24 and 48 hours. Though multiple visit root canal treatment is a safe and common treatment option, single visit endodontic treatment can also be opted as an effective alternative to two-visit treatment, especially in patients who seem to default after the first appointment.

\section{REFERENCES}

1. Claffey E. Reader Al, John N, Mike B, Joel W. Anesthetic efficacy of articaine for inferior alveolar nerve blocks in patients with irreversible pulpitis. J Endod 2004;30(8):568-71.

2. Mc Dougal RA, Delano OE, Caplan D, Sigurdsson A, Trope M. Success of an alternative for interim management of irreversible pulpitis. J Am Dent Assoc 2004;135(12):1707-12.

3. Bender IB. Reversible and irreversible painful pulpitides: diagnosis and treatment. Aust Endod J 2000;26(1):10-4.

4. Lopez-Marcos JF. Aetiology, classification \& pathogenesis of pulp and periapical disease. Med Oral Pathol Oral Cir Bucal 2004;9:58-62.

5. Ranali. Perception of Pain and Anxiety during Emergency Dental Care. Dent Anesth Res 2006:78-9.

6. Vohra F, Ahmed I, Zaki M, Shaikh A. An evaluation of etiologic factors for root canal therapy. J Pak Dent Assoc 2005;14(3):154-7.

7. Ashkenaz PJ. One-visit endodontics: A preliminary report. Dent Surv 1979;55:62-7.

8. Singh S, Garg A. Incidence of post-operative pain after single visit and multiple visit root canal treatment: A randomized controlled trial. J Conserv Dent 2012;15:323-7.

9. Oliet S. Single visit endodontics: A clinical study. J Endod 1983;9:147- 52.

10. Saleem MA, Sheikh AB, Khan MA, Atif S. Assessment of Post-obturation Pain: Single vs Two Visits Root Canal Treatment. J Pak Dent Assoc 2014;23(1):25-29.

11. Figini L, Lodi G, Gorni F, Gagliani M. Single versus multiple visits for endodontic treatment of permanent teeth. J Endod 2008;34(9):1041-7.

12. Tsesis I, Faivishevsky V, Fuss Z, Zukerman O. Flareups after endodontic treatment: a meta-analysis of literature. Journal of Endodontics 2008;34(10): 1177-81

13. Figini L, Lodi G, Gorni F, Gagliani M. Single versus multiple visits for endodontic treatment of permanent teeth. Cochrane Database of Systematic Reviews 2007; 17(4):CD005296.

14. Naito T. Single or multiple visits for endodontic treatment? Evidence-Based Dentistry 2008;9(1):24.

15. Modaresi J, Davari A, Daneskazemi A. Comparison of flare up incidence between single and two visit endodontic treatment. J Pak Dent Assoc 2007;16:134-36.

16. Albashaireh ZS, Alnegrish AS. Postobturation pain after single- and multiple-visit endodontic therapy. A prospective study. J Dent 1998;26:227-32. 